Nature

Article original

\title{
Screening phytochimique de quelques plantes médicinales ivoiriennes utilisées en pays Krobou (Agboville, Côte-d'Ivoire)
}

\author{
Koffi N'Guessan ${ }^{1}$ *, Beugré Kadja ${ }^{2}$, Guédé N. ZIRIHI ${ }^{1}$, Dossahoua Traoré ${ }^{1}$ \& Laurent AkÉ-AssI ${ }^{1}$ \\ ${ }^{1}$ Université de Cocody-Abidjan (Côte-d'Ivoire), UFR Biosciences, Laboratoire de Botanique. 22 BP 582 Abidjan 22. \\ ${ }^{2}$ Université de Cocody-Abidjan, Laboratoire de Biochimie. \\ 'Auteur pour les correspondances (E-mail : nguessankoffifr@yahoo.fr) \\ Reçu le 08-03-2007, accepté le 15-01-2009.
}

\begin{abstract}
Résumé
Une étude ethnomédicinale réalisée chez les Krobou, une ethnie du Département d'Agboville (Côte-d'Ivoire), a montré que 27 tradithérapeutes utilisent 18 espèces de la flore ivoirienne, pour combattre différentes pathologies. Diverses parties de la plante (écorces de racine et de tige, feuilles, fruits, graines et tubercules) sont utilisées pour la préparation des recettes médicamenteuses. Ces recettes, monospécifiques en général, nécessitent divers modes de préparation (décoction, expression, infusion, macération, pétrissage, pilage, pulvérisation, ramollissement, torréfaction et trituration). On note divers modes d'administration des médicaments : ablution, application locale, badigeonnage, bain de vapeur, boisson, instillations buccales, nasales, vaginales et purges. Les tests tri phytochimiques réalisés sur 3 extraits (éthérique, méthanolique et aqueux) ont révélé que les différentes drogues renferment des stérols, polyterpènes, polyphénols, flavonoïdes, tanins catéchiques, alcaloïdes et des saponosides, qui leur confèrent les propriétés thérapeutiques diverses.
\end{abstract}

Mots clefs: Agboville, Composés chimiques, Côte-d'Ivoire, Ethnomédecine, Krobou, Phytochimie.

\begin{abstract}
Phytochemical screening of some ivorian medicinal plants used by the Krobou people (Agboville, Côte-d'Ivoire)
\end{abstract}

An ethnomedicinal study carried out at Krobou, an ethnic group in the Department of Agboville (Côte-d'Ivoire) showed that 27 traditional doctors use 18 ivorian plants species to cure people to different pathologies. Various parts of the plant (barks of root and stem, sheets, fruits, seeds and tubers) are used to prepare the medicamentous receipts. These receipts which are generally monospecific, require different preparation modes (decoction, expression, infusion, soaking, kneading, pounding, crushing, softening, rousting and trituration). We note several administration modes of those medicines : washing, application on the skin, painting, steam bath, drinking, oral, nasal, vaginal instillations and purges. The tri phytochemical tests of three extracts (etheric, methanolic and aqueous) revealed that the different drugs contain sterols, polyterpenes, polyphenols, flavonoids, tannins, alkaloids and saponosides which give these medicines several healing properties.

Keywords: Agboville, chemical Components, Côte-d'Ivoire, Ethnomedicine, Krobou, Phytochemistry 


\section{Introduction}

L'utilisation des plantes, à des fins thérapeutiques, est rapportée dans les littératures antiques arabe, chinoise, égyptienne, hindou, grecque, romaine (Anonyme, 1974). En Afrique, le pouvoir thérapeutique des plantes était connu par nos ancêtres et nos parents de façon empirique (Nacoulma, 1996). Ainsi on ignorait tout de la composition chimique des médicaments utilisés tous les jours par de nombreuses populations, pour les soins de santé. Pour parvenir à une amélioration de cette médecine africaine, plusieurs investigations phytochimiques ont été faites, afin d'apporter une justification scientifique quant à l'utilisation traditionnelle des plantes médicinales. Guédé-Guina (1975) in Zirihi (2006) a mené une étude qui a débouché sur la découverte de la mansonine, un principe bioactif, extrait de Mansonia altissima (A. Chev.) A. Chev. var. altissima. En plus de l'aspect inventaire botanique, Neuwinger (1996) a consacré une partie de ses travaux à l'étude chimique et toxicologique de 305 espèces de plantes, en provenance de divers pays d'Afrique. Les travaux de Nacoulma (1996) ont porté sur l'inventaire et la composition chimique des différents organes de 391 espèces de plantes issues du Burkina Faso. Kamanzi (2002) a réalisé d'importants travaux d'ordre pharmacologique et phytochimique sur des plantes médicinales de la Côte-d'Ivoire. Des essais biologiques ont été menés sur 104 espèces de plantes médicinales. Une évaluation des activités antibactérienne, antifongique, antipaludique, antitrypanosome, a été faite ; la cytotoxité a été étudiée. Concernant les investigations phytochimiques, l'auteur a recherché les principes bioactifs des plantes étudiées ; cinq (05) isoflavonoïdes prénulés ont été isolés parmi lesquels la vogéline $A$, la vogéline $B$ et la vogéline $C$, n'avaient jamais été décrites. Koné (2005) a rapporté, dans son étude sur 135 extraits bruts végétaux, en provenance de 101 espèces de plantes médicinales de la région de Ferkessédougou, que $49 \%$ des plantes ont montré une activité anthelminthique in vitro sur Haemonchus contortus; les extraits bruts éthanoliques ont exercé une activité larvicide. Deux flavones nouvelles (l'Isosakuranétine-7-sulfate et la naningénine-7-sulfate) ont été isolées de Parinari excelsa Sabine (Chrysobalanaceae). Les travaux de Zirihi et al. (2005) ont montré que l'activité antiplamodiale de Funtumia elastica était due à un alcaloïde, I'holarrhesine. Dans une étude chez les Bété du Département d'Issia, Zirihi (2006) a testé l'activité antiplasmodiale de 39 espèces de plantes. Des études bio-guidées réalisées sur les extraits éthanoliques de Rauvolfia vomitoria ont permis d'isoler un alcaloïde dénommé rauvomitine. A travers des travaux à caractère phytochimique, Zirihi et al. (2007) ont indiqué que Zanthoxylum gilletii exerce son activité antipaludique grâce à un alcaloïde, la dihydronitidine.

Notre objectif est de faire une étude tri phytochimique de 3 extraits (éthérique, méthanolique et aqueux), en provenance de plusieurs drogues. II s'agira de caractériser les groupes chimiques qui permettront d'expliquer les effets thérapeutiques des plantes testées.

\section{Matériel et méthodes}

\subsection{Présentation du milieu d'étude}

Nous avons mené nos investigations ethnomédicinales dans 3 villages du Département d'Agboville: Aboudé-Kouassikro, AboudéMandéké et Oress-Krobou (Figs. 1 et 2). Situés à environ $120 \mathrm{~km}$ d'Abidjan, ces villages font partie du Sud de la Côte-d'Ivoire, caractérisé par une forêt dense humide semi-décidue actuellement dégradée (N'Guessan, 1995). La population autochtone est composée de Krobou, une ethnie de 18.000 âmes. II existe une petite communauté d'allochtones en provenance de plusieurs régions de la Côte-d'Ivoire et d'allogènes originaires de la sous région Ouest africaine. 


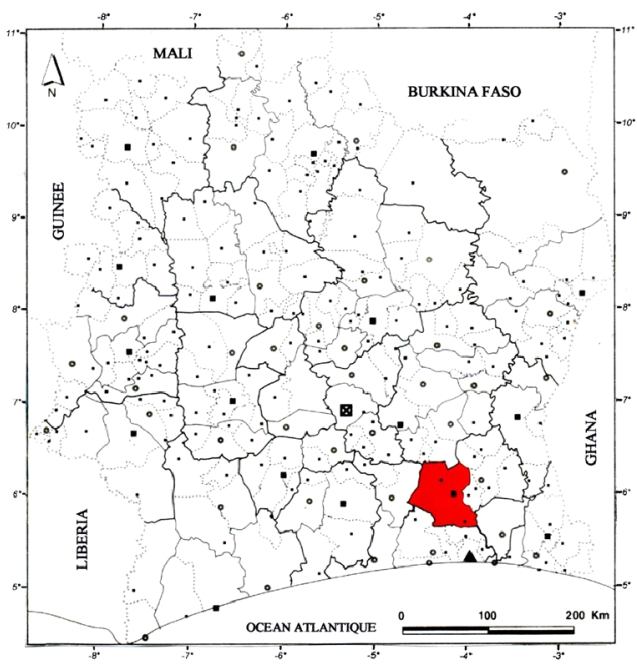

LEGENDE

Départementd'Agboville

Echelle : $1 / 3.500 .000$

Figure 1: Situation géographique du Département d'Agboville, en Côte-d'Ivoire (CEDA, 2001, modifiée par N'Guessan)

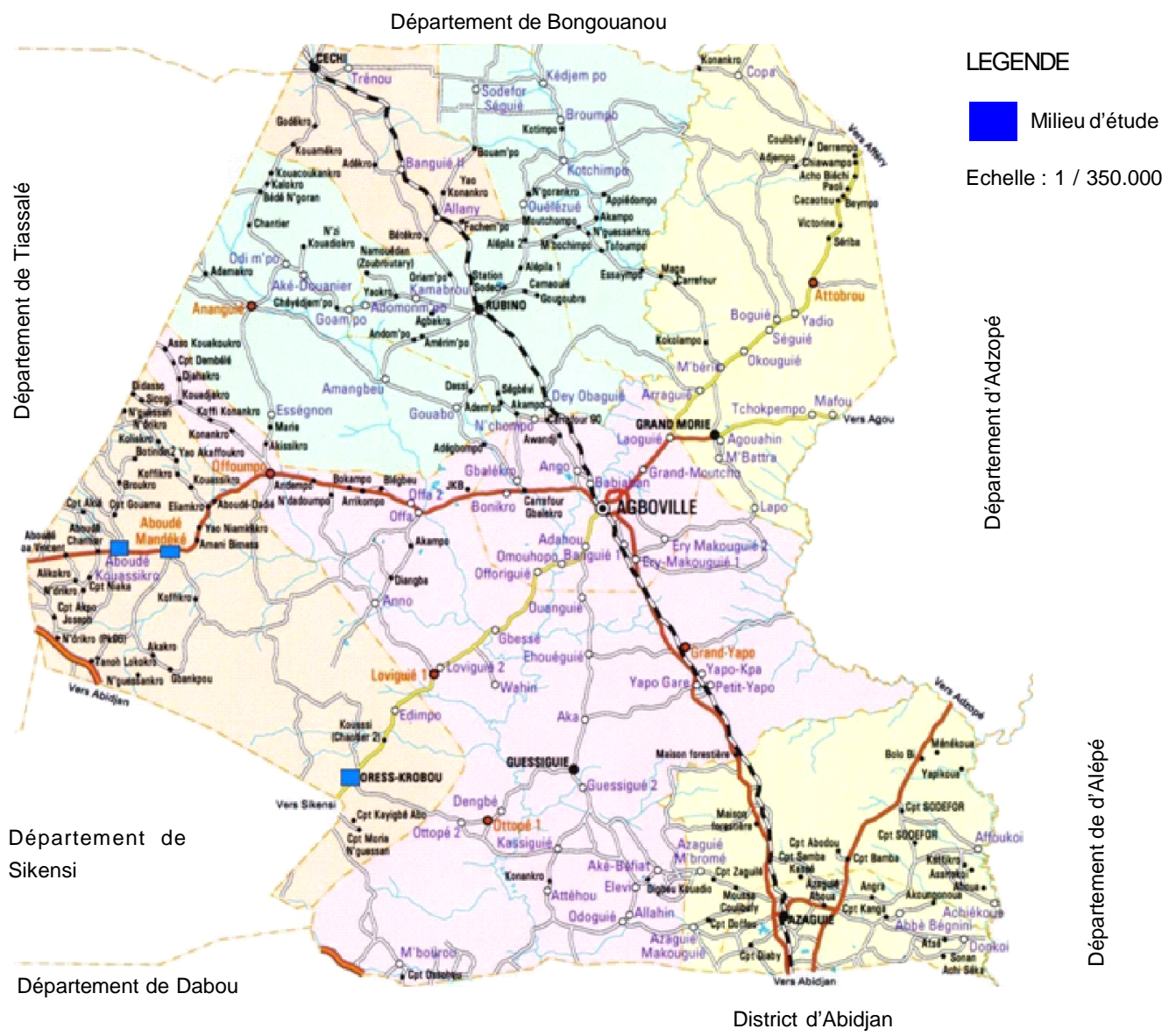

Figure 2: Situation du milieu d'étude dans le Département d'Agboville, BNETD (2004), Modifiée par N'Guessan

Sci. Nat. Vol. 6 N¹: 1 - $15(2009)$ 


\subsection{Matériel}

\subsubsection{Matériel technique}

Pour l'étude ethnomédicinale, un matériel classique a permis d'avoir accès aux plantes et de faire des prélèvements d'échantillons. En rapport avec le screning phytochimique, nous avons utilisé une étuve à $33^{\circ} \mathrm{C}$, pour sécher les échantillons devenus humides, avant leur pulvérisation grâce à une broyeuse électrique. Nous avons employé une balance électrique pour les différentes pesées, un bain-marie à $37^{\circ} \mathrm{C}$. Un bain de sable a servi à faire évaporer les extraits dans des capsules en porcelaine. Nous disposions d'une chauffe eau. Ce matériel comportait également des spatules, pour le prélèvement des poudres de drogue, du coton hydrophile utilisé comme filtre, une hotte de protection contre les poudres éjectées lors de la pulvérisation des drogues, une baguette de trituration et des pinces.

\subsubsection{Matériel végétal}

L'étude tri phytochimique a nécessité un matériel représenté par diverses drogues (écorces de racine et de tige, feuilles, fruits, graines et tubercules) qui ont été pulvérisées et utilisées pour la préparation des extraits éthérique, méthanolique et aqueux.

\subsubsection{Solvants}

Les extraits éthérique, méthanolique et aqueux ont été obtenus par extractions successives avec des solvants, en fonction de l'ordre croissant de leur polarité. Dans cet ordre, nous avons employé l'éther de pétrole, le méthanol et l'eau distillée. Les tests de détection des groupes composés ont porté sur ces 3 types d'extraits bruts.

\subsubsection{Réactifs}

Le screening phytochimique a nécessité divers réactifs. La recherche des tanins catéchiques a été possible grâce au réactif de Stiasny et à l'acétate de sodium. Pour la caractérisation des tanins galliques, nous avons employé le réactif de Stiasny, l'acétate de sodium et du chlorure ferrique. L'anhydride acétique et l'acide sulfurique concentré ont été nécessaires à la recherche des stérols et polyterpènes. L'alcool chlorhydrique dilué 2 fois, les copeaux de magnésium et l'alcool isoamylique ont été utilisés pour rechercher les flavonoïdes. La solution alcoolique de chlorure ferrique à $2 \%$ a permis la caractérisation des polyphénols. Le réactif de Bornstraëgen, le chloroforme, l'ammoniaque dilué 2 fois et l'acide chlorhydrique ont permis de rechercher les substances quinoniques. Nous avons caractérisé les alcaloïdes à partir de l'alcool à $60^{\circ}$, du réactif de Burchard (réactif iodo-ioduré) et du réactif de Dragendorff (réactif à l'iodobismuthate de potassium).

\subsection{Méthodes}

L'enquête ethnomédicinale a été réalisée auprès de 27 tradithérapeutes (11 hommes et 16 femmes) parmi les autochtones. L'approche utilisée est l'entretien semi-direct. Les échantillons récoltés ont été identifiés au Centre National de Floristique (C. N. F.).

Les différentes drogues ont été récoltées puis nettoyées et séchées à l'air libre pendant deux semaines. Elles ont été pulvérisées à l'aide d'un broyeur électrique; pour chaque drogue, nous avons retenu $200 \mathrm{~g}$ de poudre fine. Pour mener l'étude tri phytochimique, nous avons réalisé, sur chaque poudre, 3 extractions, selon le protocole mis au point par Nemlin et Brunel (1995). Les extraits bruts ont été obtenus par extractions successives, avec des solvants de polarités croissantes. Dans cet ordre, nous avons utilisé l'éther de pétrole, le méthanol et l'eau. Pour l'extraction à l'éther de pétrole, nous avons dissout $20 \mathrm{~g}$ de poudre obtenue de chaque drogue dans $60 \mathrm{ml}$ d'éther de pétrole. L'ensemble a été homogénéisé par agitation manuelle pendant $10 \mathrm{~min}$. La mixture a été ensuite filtrée. Le filtrat obtenu a été nommé filtrat éthéré 1 . Sur les marcs résiduels, nous avons ajouté $60 \mathrm{ml}$ d'éther de pétrole ; après $10 \mathrm{~min}$ d'agitation puis filtration, nous avons obtenu le filtrat éthéré 2 . La même opération a permis d'obtenir le filtrat éthéré 3 . Ces 3 filtrats ont été regroupés et concentrés à $25 \mathrm{ml}$ sur un bain de sable. Cette série d'opérations a conduit à une solution concentrée que nous avons appelée extrait éthérique. Après épuisement à l'éther de pétrole, le marc résiduel a été séché. La poudre obtenue a été récupérée dans $60 \mathrm{ml}$ de méthanol. Dix (10) min d'homogénéisation par agitation manuelle ont permis d'obtenir le filtrat méthanolique 1. La même opération a été 
reprise et elle a donné le filtrat méthanolique 2 . Les 2 filtrats méthanoliques réunis ont été filtrés et concentrés à $25 \mathrm{ml}$, au bain de sable, pour donner l'extrait méthanolique. Pour préparer l'extrait aqueux, nous avons infusé $5 \mathrm{~g}$ de la poudre sèche de chaque drogue dans $50 \mathrm{ml}$ d'eau distillée, pendant $15 \mathrm{~min}$. L'infusé a été filtré pour produire l'extrait aqueux.

Nous avons caractérisé les différents groupes chimiques en nous référant aux techniques décrites dans les travaux de Ronchetti et Russo (1971), Hegnauer (1973), Wagner (1983), Békro et al. (2007).

Les stérols et les polyterpènes ont été recherchés par la réaction de Liebermann. Cinq (5) ml de chacun des trois extraits ont été évaporés sur bain de sable. Le résidu est dissout à chaud dans $1 \mathrm{ml}$ d'anhydride acétique ; nous avons ajouté $0,5 \mathrm{ml}$ d'acide sulfurique concentré au triturât. L'apparition, à l'interphase, d'un anneau pourpre ou violet, virant au bleu puis au vert, a indiqué une réaction positive.

La réaction au chlorure ferrique $\left(\mathrm{FeCl}_{3}\right)$ a permis de caractériser les polyphénols. A $2 \mathrm{ml}$ de chaque extrait (éthérique, méthanolique et aqueux), nous avons ajouté une goutte de solution alcoolique de chlorure ferrique à $2 \%$. L'apparition d'une coloration bleu-noirâtre ou verte plus ou moins foncée fut le signe de la présence de polyphénols.

Les flavonoïdes ont été recherchés par la réaction à la cyanidine. Deux (2) $\mathrm{ml}$ de chaque extrait ont été évaporés et le résidu a été repris dans $5 \mathrm{ml}$ d'alcool chlorhydrique dilué 2 fois. En ajoutant 2 à 3 copeaux de magnésium, il y a un dégagement de chaleur puis une coloration roseorangé ou violacée. L'addition de 3 gouttes d'alcool isoamylique a intensifié cette coloration qui a confirmé la présence de flavonoïdes.

La recherche des tanins catéchiques s'est réalisée à partir du réactif de Stiasny. Cinq (5) $\mathrm{ml}$ de chaque extrait ont été évaporés à sec. Après ajout de $15 \mathrm{ml}$ du réactif de Stiasny au résidu, le mélange a été maintenu au bain-marie à $80^{\circ} \mathrm{C}$ pendant $30 \mathrm{~min}$. L'observation d'un précipité en gros flocons a caractérisé les tanins catéchiques. Pour les tanins galliques, nous avons filtré la solution précédente. Le filtrat est recueilli et saturé d'acétate de sodium. L'addition de 3 gouttes de $\mathrm{FeCl}_{3}$ provoquerait l'apparition d'une coloration bleu-noir intense, signe de la présence de tanins galliques.

Les substances quinoniques ont été recherchées à partir du réactif de Bornstraëgen. Deux (2) $\mathrm{ml}$ de chacun des 3 extraits ont été évaporés à sec. Le résidu est trituré dans $5 \mathrm{ml}$ d'acide chlorhydrique au $1 / 5$. Le triturât est versé dans un tube à essais. Le triturât est porté ensuite au bain-marie pendant $30 \mathrm{~min}$. Après refroidissement, il est extrait par $20 \mathrm{ml}$ de chloroforme. L'ammoniaque dilué 2 fois $(0,5 \mathrm{ml})$ a été ajouté à la solution chloroformique. Une coloration rouge ou violette constituait le signe de la présence de quinones.

Les alcaloïdes ont été caractérisés à partir des réactifs de Burchard (réactif iodo-ioduré) et de Dragendorff (réactif à l'iodo-bismuthate de potassium). Six (6) $\mathrm{ml}$ de chaque solution ont été évaporés à sec. Le résidu est repris par $6 \mathrm{ml}$ d'alcool à $60^{\circ}$. L'addition de 2 gouttes du réactif de Dragendorff sur la solution alcoolique provoquait un précipité ou une coloration orangée. L'ajout de 2 gouttes du réactif de Burchard sur la solution alcoolique provoquait un précipité de coloration brun-rougeâtre et indiquait une réaction positive.

Pour rechercher les saponosides, nous avons versé, dans un tube à essais, $10 \mathrm{ml}$ de l'extrait total aqueux. Le tube était agité pendant $15 \mathrm{~s}$ puis laissé au repos durant $15 \mathrm{~min}$. Une hauteur de mousse persistante, supérieure à $1 \mathrm{~cm}$ indiquait la présence de saponosides.

\section{Résultats et discussion}

\section{1. Étude ethnomédicinale}

\subsubsection{Plantes recensées et leurs indications thérapeutiques}

Les résultats concernant les plantes recensées et leurs indications thérapeutiques sont consignés dans le tableau 1. 
Tableau 1: Plantes et indications thérapeutiques

Signification des abréviations et sigles : Alc: Alcoolique ; Badig: Badigeonnage ; Citn: Citron; ER: Ecorce de racine ; ET: Ecorce de tige ; Exp : Expression ; Fe : Feuille ; Fr : Fruit ; Ge : Graine ; Instil : Instillations ; KTK : Koutoukou ; Mcré : Macéré ; Pge : Pétrissage ; Pulv: Pulvérisation; Ram : ramollissement ; RF : Rameau feuillé ; TC : tubercule caulinaire; Tritn: Trituration.

\begin{tabular}{|c|c|c|c|c|c|}
\hline Plantes & $\begin{array}{c}\text { Affections, } \\
\text { Symptômes, Effets } \\
\text { thérapeutiques et } \\
\text { phénomènes } \\
\text { morbides }\end{array}$ & 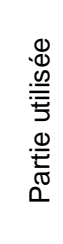 & 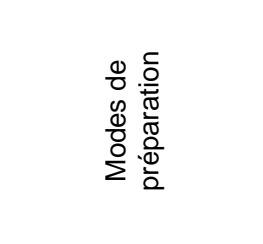 & 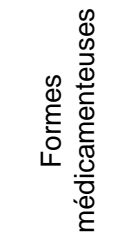 & \& \\
\hline $\begin{array}{l}\text { Aframomum } \\
\text { melegueta }\end{array}$ & Métrorragie & $\begin{array}{l}\mathrm{Fe} \\
\mathrm{Ge}\end{array}$ & $\begin{array}{l}\text { Torréfaction, Pulv + } \\
\text { eau }\end{array}$ & Poudre & Purge \\
\hline \multirow{3}{*}{$\begin{array}{l}\text { Ageratum } \\
\text { conyzoides }\end{array}$} & $\begin{array}{l}\text { Accouchement } \\
\text { facile }\end{array}$ & $\mathrm{Fe}$ & Expression & Extrait & Instil nasales \\
\hline & Migraine & $\mathrm{Fe}$ & Pilage + eau & Pâte & Instil nasales \\
\hline & Paludisme & $\mathrm{Fe}$ & Décoction & Décocté & Ablution \\
\hline \multirow{5}{*}{ Boerhvia diffusa } & Asthme & $\mathrm{Fe}$ & Infusion & Infusé & Boisson \\
\hline & Choléra & $\mathrm{Fe}$ & $\begin{array}{l}\text { Pétrissage +eau } \\
\text { Décoction }\end{array}$ & $\begin{array}{l}\text { Pâte } \\
\text { Décocté }\end{array}$ & $\begin{array}{l}\text { Purge } \\
\text { Boisson }\end{array}$ \\
\hline & $\begin{array}{l}\text { Hémorragie après } \\
\text { délivrance }\end{array}$ & $\begin{array}{l}\mathrm{Fe} \\
\mathrm{TC}\end{array}$ & $\begin{array}{l}\text { Trituration } \\
\text { Râpage }\end{array}$ & $\begin{array}{l}\text { Extrait } \\
\text { Extrait }\end{array}$ & $\begin{array}{l}\text { Instillations } \\
\text { vaginales }\end{array}$ \\
\hline & Paludisme & $\mathrm{Fe}$ & Décoction & Décocté & Boisson \\
\hline & Zona & $\mathrm{Fe}$ & Trituration +eau & Pâte & Badig, Purge \\
\hline \multirow{2}{*}{$\begin{array}{l}\text { Capsicum } \\
\text { frutescens }\end{array}$} & Rhumatisme & $\mathrm{Fr}$ & Pétrissage & Pâte & Friction \\
\hline & Stimulant laxatif & $\mathrm{Fr}$ & Pétrissage + eau & Pâte & Purge \\
\hline \multirow{3}{*}{$\begin{array}{l}\text { Chromolaena } \\
\text { odorata }\end{array}$} & Diabète & $\mathrm{Fe}$ & Décoction & Décocté & Boisson \\
\hline & Diarrhée & $\mathrm{Fe}$ & Pétrissage + eau & Pâte & Purge \\
\hline & Paludisme & $\mathrm{Fe}$ & Décoction & Décocté & Boisson \\
\hline $\begin{array}{l}\text { Coffea } \\
\text { canephora }\end{array}$ & Hypertension & $\mathrm{Fe}$ & Décoction & Décocté & Boisson \\
\hline \multirow[t]{2}{*}{ Cola nitida } & $\begin{array}{l}\text { Accouchement } \\
\text { facile }\end{array}$ & ET & Ramollissement & Extrait & Instil buccales \\
\hline & Migraine & ET & Pétrissage + eau & Pâte & Instil nasales \\
\hline Cordia vignei & Paludisme & $\mathrm{Fe}$ & Décoction & Décocté & Boisson \\
\hline \multirow{4}{*}{ Ficus exasperata } & $\begin{array}{l}\text { Accouchement } \\
\text { facile }\end{array}$ & $\mathrm{Fe}$ & Ram, exp & Extrait & Instil buccales \\
\hline & Entéralgie & $\mathrm{Fe}$ & Pge + jus citn & Pâte & Boisson \\
\hline & Fièvre typhoïde & $\mathrm{Fe}$ & Décoction & Décocté & Boisson \\
\hline & Paludisme & ET & $\begin{array}{l}\text { Macération } \\
\text { Pétrissage + eau }\end{array}$ & $\begin{array}{l}\text { Macéré } \\
\text { Pâte }\end{array}$ & $\begin{array}{l}\text { Boisson } \\
\text { Purge }\end{array}$ \\
\hline \multirow{2}{*}{ Glyphaea brevis } & Fièvre & $\mathrm{Fe}$ & Décoction & Décocté & Boisson \\
\hline & Stérilité féminine & $\mathrm{Fe}$ & Décoction & Décocté & Boisson \\
\hline \multirow{2}{*}{$\begin{array}{l}\text { Monodora } \\
\text { myristica }\end{array}$} & Céphalées & $\mathrm{Ge}$ & Pétrissage + eau & Pâte & Instil nasales \\
\hline & Vulnéraires & $\mathrm{Ge}$ & Pétrissage & Pâte & Pansement \\
\hline \multirow{3}{*}{$\begin{array}{l}\text { Ocimum } \\
\text { gratissimum }\end{array}$} & Epistaxis & $\mathrm{Fe}$ & Ram, exp & Extrait & Instil nasales \\
\hline & Hypertension & $\mathrm{Fe}$ & Décoction & Décocté & Boisson \\
\hline & Paludisme & $\mathrm{Fe}$ & $\begin{array}{l}\text { Décoction } \\
\text { Pétrissage + eau }\end{array}$ & $\begin{array}{l}\text { Décocté } \\
\text { Pâte }\end{array}$ & $\begin{array}{l}\text { Bain vapeur } \\
\text { Purge }\end{array}$ \\
\hline \multirow{3}{*}{ Paullinia pinnata } & Fièvre & $\mathrm{Fe}$ & Décoction & Décocté & Boisson \\
\hline & hypertension & $\mathrm{Fe}$ & Décoction & Décocté & Boisson \\
\hline & Contraception & $\mathrm{Fe}$ & Décoction & Décocté & Boisson \\
\hline $\begin{array}{l}\text { Petersianthus } \\
\text { macrocarpus }\end{array}$ & Choléra & ET & Ramollissement & Extrait & Purge \\
\hline
\end{tabular}

Sci. Nat. Vol. 6 N¹: 1 - 15 (2009) 
Tableau 1 (suite et fin) : Plantes et indications thérapeutiques

\begin{tabular}{|c|c|c|c|c|c|}
\hline Plantes & $\begin{array}{c}\text { Affections, } \\
\text { Symptômes, Effets } \\
\text { thérapeutiques et } \\
\text { phénomènes } \\
\text { morbides }\end{array}$ & 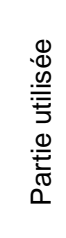 & 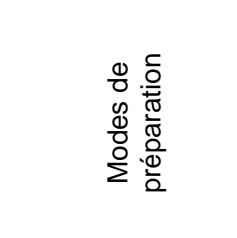 & 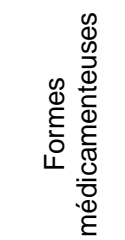 & 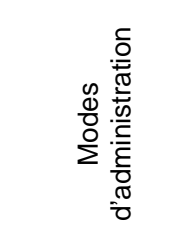 \\
\hline \multirow{5}{*}{$\begin{array}{l}\text { Phyllanthus } \\
\text { amarus }\end{array}$} & $\begin{array}{l}\text { Accouchement } \\
\text { Facile }\end{array}$ & RF & Pétrissage +eau & Pâte & Purge \\
\hline & Fièvre & RF & Décoction & Décocté & Boisson \\
\hline & Hypertension & RF & Décoction & Décocté & Boisson \\
\hline & Paludisme & RF & Décoction & Décocté & Boisson \\
\hline & Ulcères gastriques & RF & Tritn + KTK & Mcré alc & Boisson \\
\hline \multirow{4}{*}{$\begin{array}{l}\text { Rauvolfia } \\
\text { vomitoria }\end{array}$} & $\begin{array}{l}\text { Accouchement } \\
\text { facile }\end{array}$ & $\mathrm{Fe}$ & Ram, exp & Extrait & Instil buccales \\
\hline & Epilepsie & $\begin{array}{l}\mathrm{Fe} \\
\mathrm{ER}\end{array}$ & Décoction & Décocté & $\begin{array}{l}\text { Bain vapeur } \\
\text { Boisson }\end{array}$ \\
\hline & $\begin{array}{l}\text { Oedèmes des } \\
\text { pieds }\end{array}$ & ET & Pétrissage + eau & Pâte & Purge \\
\hline & Paludisme & $\mathrm{Fe}$ & Décoction & Décocté & Boisson \\
\hline $\begin{array}{l}\text { Sterculia } \\
\text { tragacantha }\end{array}$ & Asthme & $\mathrm{Fe}$ & Décoction & Décocté & Boisson \\
\hline Trema orientalis & Hypertension & $\mathrm{Fe}$ & Décoction & Décocté & Boisson \\
\hline
\end{tabular}

\subsubsection{Caractéristiques ethnomédicinales}

\subsubsection{Parties de la plante utilisées comme drogues}

Diverses parties de la plante (écorces de racine et de tige, feuilles, fruits, graines, rameaux feuillés et tubercules) sont utilisées pour les préparations médicamenteuses. Les feuilles $(63,52 \%)$ sont majoritairement sollicitées. Ce résultat est identique à celui de Zirihi (1991) qui a montré que les feuilles étaient majoritairement employées dans $64,49 \%$ des cas, chez les Bété d'Issia (Côte-d'Ivoire). Il est proche de celui établi par Adjanohoun et Aké Assi (1979) qui ont indiqué que les feuilles étaient sollicitées, majoritairement, dans $59,10 \%$ des cas. Vangah (1986) a mené son étude sur les plantes médicinales, dans la région littorale de la Côte-d'Ivoire. Elle a montré que les ethnies akans de cette région emploient majoritairement les feuilles $(50,90 \%)$. Le prélèvement intense des feuilles ne présente pas de danger pour la plante. Selon Poffenberger et al. (1992) cité par Ouattara (2006), le prélèvement de $50 \%$ des feuilles d'un arbre n'affecte pas de façon significative sa survie.

\subsubsection{Modes de préparation des médicaments}

Nous distinguons divers modes de préparations médicamenteuses (décoction, expression, infusion, macération, pétrissage, pilage, pulvérisation, ramollissement, râpage, torréfaction et trituration). La décoction est le mode de préparation le plus sollicité $(42,30 \%)$. Ce résultat est proche de celui établi par Adjanohoun et Aké Assi (1979) qui indiquent que la décoction est majoritairement utilisée dans $32,94 \%$ des cas.

\subsubsection{Modes d'administration des médicaments}

On note divers modes d'administration des médicaments : ablution, badigeonnage, bain de vapeur, boisson, frictions, instillations buccales, instillations nasales, instillations vaginales, pansement et purge. La boisson (48,97\%) est majoritairement sollicitée. Ce résultat est proche de celui établi par Ouattara (2006), Adjanohoun et Aké Assi (1979) qui ont indiqué dans leur étude que le mode d'administration le plus sollicité est la boisson qui intervient, respectivement dans 32,35 et $27,06 \%$ des cas. 


\subsubsection{Affections traitées}

Le paludisme a été le plus souvent cité, parmi les 24 affections traitées ( 7 fois sur 40 ), ce qui représente $17,50 \%$ des cas. Ce résultat s'accorde avec celui de Ouattara (2006) et Vangah (1986) qui indiquent que le paludisme représente, respectivement, $17,78 \%$ et $18,60 \%$ des pathologies traitées. Nos résultats ainsi que ceux de Ouattara (2006) et Vangah (1986) traduisent le profil sanitaire de la Côte-d'Ivoire. Le paludisme constitue, en effet, la première cause des consultations générales (P.N.L.P., 2005).

\section{2. Étude tri phytochimique}

3.2.1. Tests de détection des groupes chimiques responsables des effets thérapeutiques

Les tests de caractérisation tri phytochimique, réalisés sur les trois fractions de substances naturelles, ont donné les résultats que nous présentons dans le tableau 2.

Tableau 2 : Screening phytochimique de 18 échantillons de plantes

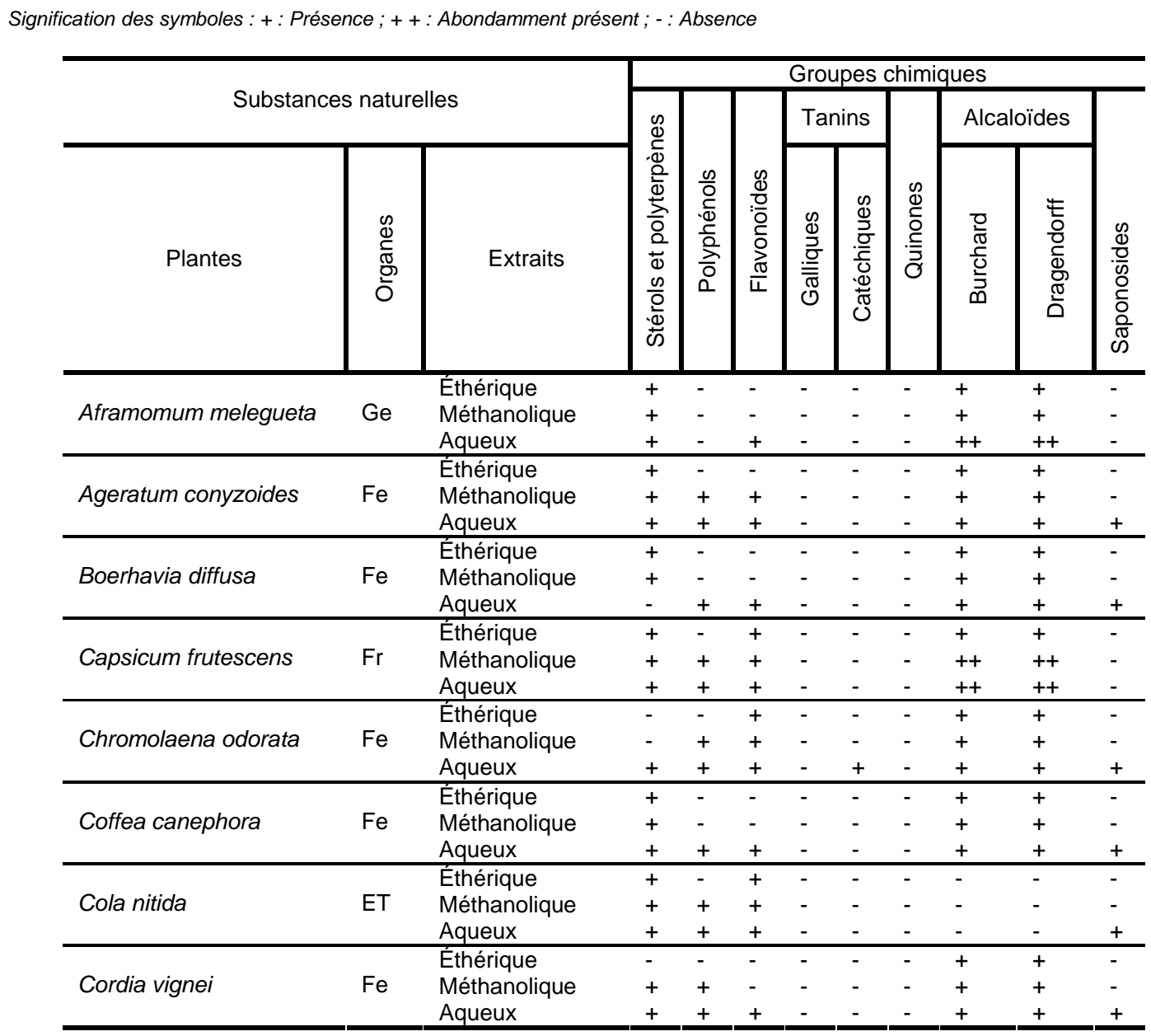


Tableau 2 (suite et fin) : Screening phytochimique de 18 échantillons de plantes

\begin{tabular}{|c|c|c|c|c|c|c|c|c|c|c|c|}
\hline & & & & & & Grou & pes & him & ues & & \\
\hline Sunstal & Malur & & & & & Tan & & & Alca & ïdes & \\
\hline Plantes & 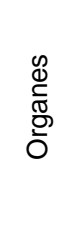 & Extraits & $\begin{array}{l}\frac{2}{0} \\
\frac{1}{2} \\
\frac{0}{0} \\
\frac{1}{0} \\
\frac{0}{0} \\
\frac{0}{0} \\
\dot{\omega}\end{array}$ & $\begin{array}{l}\frac{0}{0} \\
0 \\
\frac{0}{0} \\
\frac{1}{2} \\
\frac{2}{0} \\
0\end{array}$ & $\begin{array}{l}\mathscr{0} \\
\frac{0}{0} \\
: 0 \\
\frac{0}{0} \\
\frac{0}{0} \\
\frac{\pi}{4}\end{array}$ & 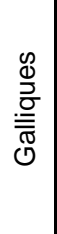 & 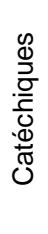 & 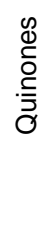 & 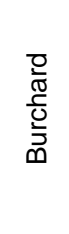 & $\begin{array}{l}\frac{\bar{z}}{0} \\
\overline{0} \\
\frac{0}{0} \\
\frac{0}{\pi} \\
\overline{0}\end{array}$ & 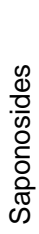 \\
\hline & & Éthérique & + & - & - & - & - & - & + & + & - \\
\hline Ficus exasperata & $\mathrm{Fe}$ & Méthanolique & + & - & - & - & - & - & + & + & - \\
\hline & & Aqueux & + & + & + & - & - & - & + & + & - \\
\hline & & Éthérique & + & - & - & - & - & - & - & - & - \\
\hline Glyphaea brevis & $\mathrm{Fe}$ & Méthanolique & + & - & - & - & - & - & + & + & - \\
\hline & & Aqueux & + & - & + & - & - & - & + & + & - \\
\hline & & Éthérique & + & - & - & - & - & - & - & - & - \\
\hline Monodora myristica & Ge & Méthanolique & + & - & + & - & - & - & + & + & - \\
\hline & & Aqueux & + & - & $\begin{array}{l}+ \\
+ \\
\end{array}$ & - & - & - & ++ & ++ & - \\
\hline & & Éthérique & + & - & - & - & - & - & + & + & - \\
\hline Ocimum gratissimum & $\mathrm{Fe}$ & Méthanolique & + & + & - & - & - & - & + & + & - \\
\hline & & Aqueux & + & + & + & - & + & - & ++ & ++ & - \\
\hline & & Éthérique & + & - & + & - & - & - & + & + & - \\
\hline Paullinia pinnata & $\mathrm{Fe}$ & Méthanolique & + & + & + & - & - & - & + & + & - \\
\hline & & Aqueux & + & + & + & - & + & - & + & + & + \\
\hline & & Éthérique & + & + & + & - & - & - & + & + & - \\
\hline $\begin{array}{l}\text { Petersianthus } \\
\text { macrocarpus }\end{array}$ & ET & Méthanolique & + & + & + & - & - & - & + & + & - \\
\hline & & Aqueux & - & + & + & - & + & - & + & + & + \\
\hline & & Éthérique & + & - & + & - & - & - & + & + & - \\
\hline Phyllanthus amarus & $\mathrm{Fe}$ & Méthanolique & + & + & + & - & - & - & + & + & - \\
\hline & & Aqueux & - & + & + & - & + & - & + & + & - \\
\hline & & Éthérique & + & - & - & - & - & - & + & + & - \\
\hline Rauvolfia vomitoria & $\mathrm{Fe}$ & Méthanolique & + & - & - & - & - & - & + & + & - \\
\hline & & Aqueux & + & - & - & - & - & - & + & + & + \\
\hline & & Éthérique & + & - & - & - & - & - & + & + & - \\
\hline Sterculia tragacantha & $\mathrm{Fe}$ & Méthanolique & + & + & + & - & - & - & + & + & - \\
\hline & & Aqueux & + & + & + & - & - & - & + & + & + \\
\hline & & Éthérique & + & - & - & - & - & - & + & + & - \\
\hline Trema orientalis & $\mathrm{Fe}$ & Méthanolique & + & + & + & - & - & - & + & + & - \\
\hline & & Aqueux & + & + & + & - & + & - & + & + & - \\
\hline
\end{tabular}

D'un test à l'autre, nous notons l'absence de quinones, dans les 3 extraits et pour tous les échantillons. C'est aussi le cas des tanins galliques. Les alcaloïdes sont présents dans tous les extraits et chez tous les échantillons, sauf chez Cola nitida (Sterculiaceae). La réaction de Liebermann est positive dans toutes les drogues testées, ce qui indique que toutes les substances végétales considérées renferment des stérols et des polyterpènes. S'agissant des autres métabolites secondaires (polyphénols, flavonoïdes, tanins catéchiques, saponosides), les résultats varient d'un extrait à l'autre et d'un échantillon à l'autre. 
3.2.2. Bases tri phytochimiques de l'utilisation thérapeutique de 18 espèces de plantes

\section{Aframomum melegueta K. Schum. (Zingiberaceae)}

Les graines contiennent des stérols, polyterpènes, flavonoïdes et des alcaloïdes. L'effet antimétrorragique des graines serait le fait des flavonoïdes. Cet effet thérapeutique est rapporté par Nacoulma (1996) qui a signalé que les flavonoïdes réduiraient les hémorragies. Les stérols, les polyterpènes et les alcaloïdes permettent d'expliquer des utilisations thérapeutiques traditionnelles signalées par d'autres auteurs mais non révélées par les résultats de cette enquête. Nacoulma (1996) indique que les graines de la plante sont utilisées contre les maux de ventre du fait des alcaloïdes tropaniques, réputés contre les spasmes gastro-intestinaux. Les alcaloïdes pyrrolizidiniques exercent un effet ocytocique et sont donc utiles pour faciliter les accouchements. Les stérols et les polyterpènes expliqueraient l'effet vulnéraire signalé par Adjanohoun et Aké Assi (1979).

\section{Ageratum conyzoides L. (Asteraceae)}

Les feuilles contiennent stérols, polyterpènes, polyphénols, flavonoïdes, alcaloïdes et saponosides. Kerharo et Adam (1974) n'ont mis en évidence que des phénols et alcaloïdes; les travaux de Bouquet et Debray (1974) révèlent des tests négatifs quant aux saponosides, flavonoïdes, stérols et polyterpènes, contrairement à ce que nous avons mis en évidence. L'effet ocytocique des feuilles de la plante serait le fait des flavonoïdes de type flavanones ainsi que des alcaloïdes indoliques. L'effet contre la migraine serait le fait des alcaloïdes, probablement de type ergotamine (Frantisek et al., 1973). Les flavonoïdes réduiraient les hémorragies, ce qui explique l'emploi de la plante, contre les métrorragies. L'action antipaludique de la plante serait le fait des alcaloïdes. Les stérols, les polyterpènes, les polyphénols, et les saponosides exercent divers effets thérapeutiques que nos enquêtes ne révèlent pas mais qui sont signalés dans d'autres travaux. Nacoulma (1996) indique l'effet cicatrisant des feuilles, par le fait des saponosides. Selon Bouquet et Debray (1974), les feuilles sont utilisées pour calmer les saignements utérins, du fait des polyphénols de types coumarines qui agissent contre les hémorragies viscérales. Cette même drogue est employée comme anthelminthique (Kamanzi, 2002), par le fait des polyterpènes.

\section{Boerhavia diffusa L. (Nyctaginaceae)}

Les feuilles contiennent stérols, polyterpènes, polyphénols, flavonoïdes, alcaloïdes et saponosides. L'effet anti-asthmatique serait le fait des saponosides ainsi que des stérols et des alcaloïdes (tropaniques); responsables de la vasodilatation, les alcaloïdes tropaniques freineraient les sécrétions alvéolaires et calmeraient les crises d'asthme. Les stérols et polyterpènes ont des propriétés bactéricides, ce qui serait en faveur de l'emploi de la plante, contre le choléra et le zona. Les polyphénols de types coumarines sont utiles pour arrêter les hémorragies après délivrance. L'effet antipaludique serait le fait des alcaloïdes indoliques. Kerharo et Adam (1974) montrent que les feuilles de la plante sont employées contre les oedèmes et les maladies oculaires. Ces effets thérapeutiques sont dus à un alcaloïde amer dénommé punarnavine; la punarnavine, réputée pour traiter les maladies oculaires, provoque aussi une augmentation marquée et persistante de la pression sanguine avec une forte diurèse, par action sur l'épithélium rénal ; elle est donc indiquée contre les oedèmes. Les investigations que nous avons menées n'ont pas révélé l'action de la plante contre les oedèmes ainsi que les maladies oculaires.

\section{Capsicum frutescens $\mathrm{L}$. (Solanaceae)}

Les fruits renferment stérols, polyterpènes, polyphénols, flavonoïdes et alcaloïdes. Condiment essentiel dans la cuisine de toute l'Afrique intertropicale, le petit piment agit comme un stimulant laxatif. II renferme la vitamine $B_{2}$ qui a une action favorable sur les muqueuses et l'équilibre intestinal (Nacoulma, 1996). L'effet laxatif et anti-rhumatismal est dû à un alcaloïde, la capsaïne qui accroît le péristaltisme intestinal et agit sur les récepteurs périphériques. Efficace contre le météorisme abdominal, le petit piment favorise l'expulsion des gaz et la stimulation de la circulation sanguine, en cas de rhumatisme (Asekun et al., 2004). 


\section{Chromolaena odorata (L.) King et H. Robins (Asteraceae)}

Dans les feuilles, nous avons détecté divers composés : stérols, polyterpènes, polyphénols, flavonoïdes, alcaloïdes, tanins catéchiques et saponosides. L'effet antidiabétique serait le fait des alcaloïdes (indolizidiniques ou pyrrolidiniques). On a mis en évidence, dans les feuilles de Chromolaena odorata, un glucide complexe, l'inuline, polymère de fructose, qui exerce un effet hypoglycémiant, ce qui est en faveur de son emploi traditionnel, dans le traitement du diabète (A.C.C.T., 1989). Les polyphénols mis en évidence exercent un effet antidiarrhéique. Selon A.C.C.T. (1989), les feuilles de Chromolaena odorata contiennent des huiles essentielles qui exercent une activité contre Staphylococcus aureus et Escherichia coli, ce qui serait en faveur de son usage empirique, contre les diarrhées, d'origine bactérienne. Les feuilles de la plante contiennent des alcaloïdes (indoliques) qui ont des propriétés antipaludiques. L'effet antipaludique a été signalé par Zirihi (2006). Selon l'auteur, les feuilles de Chromolaena odorata sont riches en polysaccharides et en glyco-protéines; ces composés chimiques exercent une activité immunostimulante, en favorisant la production d'anticorps, mais surtout en renforçant l'activité des Lymphocytes $T$ qui possèdent un indice de stimulation élevé. La stimulation du système immunitaire permet de réorganiser et de renforcer les défenses naturelles de l'organisme, ce qui permet à l'individu de résister à l'agression du parasite.

\section{Coffea canephora Pierre ex Froehner (Rubiaceae)}

Les feuilles sont constituées des composés suivants : stérols, polyterpènes, polyphénols, flavonoïdes, alcaloïdes et saponosides. Chez le caféier, l'effet hypotenseur serait le fait des flavonoïdes ou des polyphénols, probablement des coumarines.

\section{Cola nitida (Vent.) Schott et Endl. (Sterculiaceae)}

Les tests tri phytochimiques effectués sur les écorces de tige ont mis en évidence les composés suivants: stérols, polyterpènes, polyphénols, flavonoïdes et saponosides. Sur le plan tri phytochimique, nos résultats diffèrent de ceux obtenus par Bouquet et Debray (1974), car dans leurs travaux, les tests de caractérisation des stérols, polyterpènes, polyphénols, flavonoïdes et saponosides se sont tous révélés négatifs. La facilitation de l'accouchement serait le fait des flavonoïdes (flavonones) qui exercent un effet ocytocique.

\section{Cordia vignei Hutch. et Dalz. (Boraginaceae)}

Les tests tri phytochimiques effectués sur les feuilles ont mis en évidence les composés suivants : stérols, polyterpènes, polyphénols, flavonoïdes, alcaloïdes et saponosides. Ce sont les alcaloïdes probablement de types indoliques qui exercent un effet antipaludique.

\section{Ficus exasperata Vahl (Moraceae)}

Les feuilles contiennent des stérols, polyterpènes, polyphénols, flavonoïdes et des alcaloïdes. Ce résultat diffère de celui de Bouquet et Debray (1974) où tous les tests sont négatifs. Ce sont les alcaloïdes indoliques qui exercent l'effet ocytocique. Les feuilles de la plante sont utilisées contre les maux de ventre du fait des alcaloïdes tropaniques, réputés contre les spasmes gastro-intestinaux. L'effet antipaludique serait dû aux alcaloïdes indoliques de type hermane. Kerharo et Bouquet (1950) ont montré dans leur étude que les feuilles de la plante exercent une action hémorroïdaire, du fait des flavonoïdes de types flavonols. Nos enquêtes ethnomédicinales n'ont pas révélé cet usage thérapeutique.

\section{Glyphaea brevis (Spreng.) Monachino (Tiliaceae)}

Les tests tri phytochimiques effectués sur les feuilles ont mis en évidence les composés suivants : stérols, polyterpènes, flavonoïdes et alcaloïdes. Ce résultat est différent de celui de Bouquet et Debray (1974) ; leur étude sur la caractérisation tri phytochimique n'a mis en évidence que des alcaloïdes. L'effet antipyrétique serait le fait des alcaloïdes isoquinoléiniques. Les feuilles de la plante contiennent de la vitamine E (Bouquet et Debray, 1974), vitamine de la fertilité. Cette substance active le complexe hypothalamo-hypophysaire qui, par ses gonadostimulines (FSH, LH), est responsable de la production des hormones ovariennes (oestrogènes, progestérone), ayant pour organe cible, l'utérus ; cette vitamine permet de lutter contre la stérilité féminine. La vitamine $E$ est également indiquée dans le cas d'atrophie des 
ovaires ou de l'utérus. Ouattara (2006) indique que les feuilles de la plante servent à combattre la toux, du fait des polyphénols de types coumarines. Nos enquêtes ethnomédicinales n'ont pas révélé cet usage thérapeutique.

\section{Monodora myristica (Gaertn.) Dunal (Annonaceae)}

Les graines contiennent les composés suivants : stérols, polyterpènes, flavonoïdes et alcaloïdes. Ce sont les alcaloïdes probablement de type ergotamine qui interviennent dans le traitement des maux de tête. Kouamé et al. (2004) ont montré que les graines de Monodora myristica (Annonaceae) ont des propriétés désinfectantes et antiseptiques dues aux huiles essentielles, notamment au $p$-Cymène, ce qui est en faveur de son utilisation traditionnelle, dans le traitement des blessures et plaies.

\section{Ocimum gratissimum L. (Lamiaceae)}

Les feuilles contiennent des stérols, polyterpènes, polyphénols, flavonoïdes, tanins catéchiques et des alcaloïdes. La composition phytochimique établie dans les travaux de A.C.C.T. (1989) se présente comme suit : stérols, polyterpènes, polyphénols, flavonoïdes, tanins catéchiques, quinones, alcaloïdes et saponosides. Nos résultats n'ont pas révélé l'existence de quinones et de saponosides. Les feuilles de la plante sont riches en flavonoïdes, principes bioactifs qui renforcent la paroi interne des vaisseaux sanguins et favorisent la disparition des varices; l'action de ces flavonoïdes sur les parois des vaisseaux sanguins aide à prévenir les troubles circulatoires ; c'est pourquoi, l'on emploie, traditionnellement, les feuilles de la plante, contre l'épistaxis. L'effet hémostatique peut être dû aux tanins catéchiques. L'action anti-hypertensive de la plante s'expliquerait par la présence de polyphénols, probablement de type coumarines. La plante renferme des alcaloïdes indoliques qui exercent un effet antipaludique (Bruneton, 1999).

\section{Paullinia pinnata L. (Sapindaceae)}

Les tests tri phytochimiques effectués sur les feuilles ont mis en évidence les composés suivants : stérols, polyterpènes, polyphénols, flavonoïdes, tanins catéchiques, alcaloïdes et saponosides. Cette composition diffère légèrement de celle établie par Nacoulma (1996) qui signale l'absence de polyphénols et de tanins catéchiques. Dans les travaux de Kerharo et Bouquet (1950), les alcaloïdes n'ont pas été mis en évidence. Les feuilles de la plante renferment des polyphénols, probablement des coumarines reconnues pour leurs effets antipyrétiques et hypotensives. Les isoflavonoïdes de Paullinia pinnata s'opposent à l'action des oestrogènes et sont cause d'infertilité, par inhibition de l'ovulation, ce qui permet de comprendre l'usage traditionnel de la plante en tant que contraceptif. Bouquet et Debray (1974) montrent que les feuilles de la plante sont employées contre les oedèmes. Cet effet thérapeutique serait dû à un alcaloïde amer dénommé punarnavine qui provoque une forte diurèse, par action sur l'épithélium rénal. Cette indication thérapeutique ne nous a pas été révélée lors de nos enquêtes ethnomédicinales.

\section{Petersianthus macrocarpus (P. Beauv.) Liben (Barringtoniaceae)}

Les tests tri phytochimiques effectués sur les écorces de tige ont mis en évidence les composés suivants : stérols, polyterpènes, polyphénols, flavonoïdes, tanins catéchiques, alcaloïdes et saponosides. Les écorces de tige de Petersianthus macrocarpus renferment des polyphénols (catéchols) et des tanins catéchiques qui confèrent aux écorces des propriétés bactéricides et expliquent ainsi leur emploi comme anticholérique.

\section{Phyllanthus amarus Schum. et Thonn. (Euphorbiaceae)}

Les feuilles renferment stérols, polyterpènes, polyphénols, flavonoïdes, tanins catéchiques et alcaloïdes. Les tests de caractérisation tri phytochimique se sont révélés négatifs dans les travaux de Bouquet et debray (1974). Les feuilles de la plante, grâce aux alcaloïdes qu'elles contiennent, contribuent au traitement des ulcères gastriques (plaies d'estomac), en réduisant la douleur et l'acidité. Chez Phyllanthus amarus, l'effet hypotenseur serait le fait des polyphénols, probablement des coumarines. La plante contient des alcaloïdes probablement de types indoliques qui exercent un effet antipaludique.

\section{Rauvolfia vomitoriaAfzel. (Apocynaceae)}

Les tests tri phytochimiques effectués sur les feuilles ont mis en évidence les composés 
suivants : stérols, polyterpènes, alcaloïdes et saponosides. Selon Kerharo et Adam (1974), les feuilles de la plante renferment des terpènes (vomifoliol), des flavonoïdes (kaempférol, astragaline) et des alcaloïdes ; cette composition est différente de celle que nous avons obtenue. Ce sont les alcaloïdes indoliques probablement de type agroclavine qui exercent l'effet ocytocique. Le traitement des oedèmes des pieds serait le fait d'un alcaloïde, la punarnavine (Kerharo \& Adam, 1974) qui provoque une augmentation marquée et persistante de la pression sanguine avec une forte diurèse, par action sur l'épithélium rénal. Il est reconnu que les racines de Rauvolfia vomitoria (Apocynaceae) renferment un alcaloïde de type réserpine qui possède des propriétés sédatives. Cette drogue est utilisée, pour traiter les états d'anxiété et les patients perturbés mentalement, ce qui peut souligner son emploi empirique, dans le traitement des crises d'épilepsie.

\section{Sterculia tragacantha Lindl. (Sterculiaceae)}

Les feuilles renferment les composés suivants: stérols, polyterpènes, polyphénols, flavonoïdes, alcaloïdes et saponosides. Tous les tests réalisés par Bouquet et Debray (1974) se sont révélés négatifs. Kerharo et Adam (1974) signalent l'absence d'alcaloïdes et de saponosides. Les feuilles de la plante sont employées, traditionnellement, pour combattre l'asthme; elles renferment un hétéroside dénommé adansonine qui agit en provoquant la chute de la pression carotidienne, ce qui entraîne une accélération du rythme, conférant ainsi à la plante une activité anti-asthmatique. Ouattara (2006) a signalé que les feuilles exercent un effet hypotenseur, du fait probablement des flavonoïdes (flavonols). Adjanohoun et Aké Assi (1979) indiquent que la plante est utilisée pour faciliter les accouchements; les flavonoïdes de type flavanones conféreraient aux feuilles des propriétés ocytociques. Ces effets thérapeutiques (hypotenseur et ocytocique) ne nous ont pas été révélés lors de nos enquêtes ethnomédicinales.

\section{Trema orientalis (L.) Blume (Ulmaceae)}

Les feuilles contiennent des stérols, polyterpènes, polyphénols, flavonoïdes, tanins catéchiques et des alcaloïdes. Ce résultat diffère de celui de Bouquet et Debray (1974) qui signalent l'absence d'alcaloïdes. L'effet hypotenseur de la plante serait le fait des polyphénols (coumarines) et des flavonoïdes (flavonones). Kerharo et Adam (1974) montrent que les feuilles exercent des effets diurétiques du fait des flavonoïdes et des effets vermifuges du fait des polyterpènes ; nos enquêtes n'ont pas révélé ces effets.

\section{Conclusion}

Les investigations ethnomédicinales menées chez les Krobou, montrent que 18 espèces végétales sont utilisées, sous différentes formes de préparations médicamenteuses, dans la lutte contre diverses pathologies (aménorrhée, asthme, céphalées, choléra, diabète, diarrhée, entéralgie, épilepsie, épistaxis, fièvre, fièvre typhoïde, hémorroïdes, hypertension artérielle, métrorragie, migraine, oedèmes, paludisme, stérilité féminine, rhumatisme, ulcère gastrique et zona). Les effets thérapeutiques sont induits par divers comopsés chimiques (alcaloïde, flavonoïdes, polyphénols, polyterpènes, saponosides, stérols et tanins catéchiques) qui constituent la base scientifique de l'utilisation thérapeutique traditionnelle des plantes étudiées. Nous souhaitons étendre cette étude tri phytochimique aux autres plantes de notre répertoire afin de justifier leur utilisation thérapeutique traditionnelle.

\section{Références citées}

A.C.C.T., 1989. Vers une pharmacopée caraïbe. Edition de l'Agence de Coopération Culturelle et Technique (A.C.C.T.), $476 \mathrm{pp}$.

Adjanohoun E. J. \& Aké Assi L., 1979. Contribution au recensement des plantes médicinales de Côte-d'Ivoire. Université d'Abidjan, Centre National de Floristique (C.N.F.), 358 pp.

Anonyme, 1974. Encyclopédie-Le Grand Médical. L'histoire de la médecine et de la chirurgie, l'avenir de la médecine, les prix Nobel. Edition Service S.A., Genève (Suisse), 397 pp.

Asekun O.T. \& Adeniyi B.A., 2004. Antimicrobial and cytotoxic activities of the fruit essential oil of Xylopia aethiopica from Nigeria. Fitoterapia, $75: 368-370$. 
Békro Y. A., Békro J. A. M., Boua B. B., TRA B. F. H. \& Ehilé E. E., 2007. Etude ethnobotanique et screening phytochimique de Caesalpinia benthamiana (Baill.) Herend. et Zarucchi (Caesalpiniaceae). Rev. Sci. Nat. Vol. 4 (2) : 217-225.

Bouquet A. \& Debray M., 1974, Plantes médicinales de Côte-d'Ivoire, Imprimerie Louis Jean, Paris (France), 232 pp.

Bruneton J., 1999. Pharmacognosie, Phytochimie et plantes médicinales. Edition Technique et Documentation, 1120 pp.

Frantisek S., Stary F. \& Jirasek V., 1976. Plantes médicinales. Edition Lidis, 247 pp.

Hegnauer R., 1973. Chemotaxonomie der Pflanzen, Bikhäuser Verlag, Basel, Suttgart, 6, $761 \mathrm{pp}$.

Kamanzi A., 2002. Plantes médicinales de Côted'Ivoire: investigations phytochimiques guidées par des essais biologiques. Thèse de Doctorat d'Etat, Université de CocodyAbidjan, UFR Biosciences, $N^{\circ}$ d'ordre : 363/ 2002, 176 pp.

Kerharo J. \& Bouquet A., 1950. Plantes médicinales et toxiques de la Côte-d'Ivoire Haute Volta. Vigot et Frères, Editeurs. Paris (VIè), 296 pp.

Kerharo J. \& Adam J.G., 1974, La pharmacopée sénégalaise traditionnelle. Plantes médicinales et toxiques, Vigot frères, Paris, 1007 pp.

Koné M. W., 2005. Potentiel des plantes médicinales de Côte-d'Ivoire dans le contrôle des haemonchoses chez les ovins. Thèse de Doctorat de l'Université de Cocody, UFR Biosciences, Laboratoire de Botanique, 202 pp.

Kouamé R.O., Coffi K., Guessend N., Séri Y., Koukoua G., Dosso M., Yao T.N., Figueredo G. \& Chalchat J.-C., 2004. Activités antibactériennes des huiles Essentielles de trois plantes aromatiques de Côte-d'Ivoire. Comptes rendus de Chimie, 7 : 1081-1086.

Nacoulma-Ouédraogo O., 1996, Plantes médicinales et pratiques médicales traditionnelles au Burkina Faso: cas du Plateau central, Thèse de Doctorat ès Sciences Naturelles, Université de Ouagadougou, (Burkina-Faso), 605 pp.
Nemlin J et Brunel J. F., 1995. Fascicule de Travaux Pratiques de Matière Médicale (3ème année). Université Nationale de Côte-d'Ivoire. Faculté de Pharmacie. Département de Pharmacognosie. Laboratoire de Phytologie, $47 \mathrm{pp}$.

Neuwinger H. D., 1996. African Ethnobotany. Poisons and Drugs. Chemistry, Pharmacology, Toxicology. Ed. Champman and Hall, Bundesrepublik Deutschland, 942 pp.

N'Guessan K., 1995, Contribution à l'étude ethnobotanique en pays krobou (Côted'Ivoire).Thèse de Doctorat de $3^{\text {ème }}$ cycle, Université Nationale de Côte-d'Ivoire, F.A.S.T. d'Abidjan, Nº d'ordre : 216/95, 557 pp.

Ouattara D., 2006. Contribution à l'inventaire des plantes médicinales significatives utilisées dans la région de Divo (sud forestier de la Côte-d'Ivoire) et à la diagnose du poivrier de Guinée: Xylopia aethiopica (Dunal) A. Rich. (Annonaceae). Thèse de Doctorat de l'Université de Cocody-Abidjan (Côte-d'Ivoire), UFR Biosciences, Laboratoire de Botanique, 184 pp.

Ronchetti F. \& Russo G., 1971. A new alkaloid from Rauvolfia vomitoria. Phytochemistry, Vol. 10 : 1385-1388.

Vangah-Manda M. O., 1986. Contribution à la connaissance des plantes médicinales utilisées par les ethnies Akans de la région littorale de la Côte-d'lvoire. Thèse de Doctorat de $3^{\text {ème }}$ Cycle, Université Nationale de Côted'Ivoire, F.A.S.T. d'Abidjan, 464 pp.

Wagner H., 1983. Drogen analyse, Dünschicht chromatographische Analyse von Arzneidrogen. Springer Verlag Berlin Heidelberg New York, 522 pp.

Zirihi G. N., 1991.Contribution au recensement, à l'identification et à la connaissance de quelques espèces végétales utilisées dans la médecine traditionnelle et la pharmacopée chez les Bété du Département d'Issia, Côted'lvoire. Thèse de Doctorat de 3ème Cycle, Université d'Abidjan, F.A.S.T., 150 pp.

Zirihi G. N., Grellier P., Guédé-Guina F., Bodo B. et Lengo M., 2005. Isolation, Characterisation and antiplasmodial activity of steroidal alkaloids from Funtumia elastica (Preuss) Stapf. Biorganic and Medicinal Chemistry Letters 15 : 2637-2640. 
Zirihi G. N., 2006. Études botanique, pharmacologique et phytochimique de quelques plantes médicinales anti-paludiques et/ou immunogènes utilisées chez les Bété du Département d'Issia, dans l'ouest de la Côted'Ivoire. Thèse de Doctorat d'Etat, Université de Cocody-Abidjan, UFR Biosciences, $126 \mathrm{p}$.
Zirihi G. N., Datté J. Y., Kra-Adou K. M. et Grellier P., 2007. Phytochemical and pharmacological studies of the alcoholic extract (MFA) of Fagara macrophylla (Oliv.) Engl. (Rutaceae) : the chemical structure of the active compound inducing antipaludic activity, Journal of Chinese Clinical Medicine, 2 (4) : 205-210. 